\title{
Сорбционное концентрирование и определение методом ГКР-спектроскопии пирена с использовани- ем алюмогелей, содержащих наночастицы серебра
}

\author{
Юрова Н.С., Захаревич А.М., Маркин А.В., Русанова Т.Ю. \\ Саратовский наџиональный исследовательский государственный университет имени Н.Г. Черны- \\ шевского, Саратов
}

Поступила в редакцию 20.06.2018 г.

DOI: https://doi.org/10.17308/sorpchrom.2018.18/569

Получены алюмогели, содержащие наночастицы (НЧ) серебра, и оценено их использование в качестве материалов для одновременного концентрирования пирена и его детектирования методом гигантского комбинационного рассеяния света (ГКР). НЧ серебра предварительно подвергали искусственной агрегации с помощью хлорида натрия. Изучены сорбционные свойства полученных алюмогелей по отношению к пирену, и показано повышение степени извлечения пирена на сорбентах, модифицированных додецилсульфатом натрия (ДДС). Оценена возможность определения пирена, выделенного на сорбенте, методом ГКР-спектроскопии.

Ключевые слова: алюмогель, наночастицы серебра, гигантское комбинационное рассеяния света, концентрирование, пирен.

\section{Solid-phase extraction and SERS detection of pyrene on alumina gel with incorporated silver nanoparticles}

\author{
Yurova N.S., Zakharevich A.M., Markin A.V., Rusanova T.Y. \\ Saratov State University, Saratov
}

A promising approach for the simultaneous pre-concentration and determination of polycyclic aromatic hydrocarbons (PAHs), one of the priority environment pollutants, can be a combination of sorption and subsequent detection by surface-enhanced Raman scattering (SERS). In this paper, materials based on alumina containing silver nanoparticles (NPs) have been obtained, as well as the possibility of modification of these materials, their sorption properties and their application for determination by the SERS have been studied. A method of sorption pre-concentration in a static mode was used, and pyrene as a representative of PAH was chosen as a model analyte. Preliminary, silver NPs were obtained by the citrate reduction method, their size was $70 \pm 20 \mathrm{~nm}$. An additional aggregation of silver NPs by sodium chloride was performed to enhance the SERS signal. Further, sorption of pyrene on the obtained alumina with embedded silver NPs was studied. However, the recovery of pyrene on this alumina gel did not exceed $68 \%$, which can be explained by the hydrophilicity of the sorbent. To enhance the recovery of hydrophobic pyrene, surface modification of the sorbent with surfactants was carried out. As surfactant, aqueous and acidic solutions of sodium dodecyl sulfate (SDS) of various concentrations were used. An increase in the recovery of pyrene on sorbents modified by SDS was observed. The optimal conditions for modifying the sorbent are the concentration of SDS $1.4 \cdot 10^{-2} \mathrm{M}$ and pure water as a solvent. Under the chosen conditions, an isotherm of sorption of pyrene on an alumina gel containing silver NPs was obtained, which obeys the Freundlich equation. Under optimal conditions, pyrene was pre-concentrated and determined directly in the solid phase by the SERS method.

Keywords: alumina gels, silver nanoparticles, surface-enhanced Raman spectroscopy, preconcentration, pyrene. 


\section{Введение}

Полициклические ароматические углеводороды (ПАУ) являются одними из приоритетных загрязнителей окружающей среды, обладают канцерогенными, мутагенными и другими токсическими свойствами, в связи с чем необходим постоянный контроль их содержания [1]. Для определения ПАУ широкое распространение получили спектроскопические [2], электрохимические, хроматографические, иммунохимические методы анализа [3]. Однако они требуют предварительного концентрирования ПАУ из объектов. В последнее время все большую популярность получают комбинированные методы анализа. Перспективным подходом для одновременного выделения веществ и их определения может стать сочетание сорбционного концентрирования с последующим детектированием методом спектроскопии гигантского комбинационного рассеяния (ГКР) света. На данный момент спектроскопия ГКР активно исследуется как перспективный метод для создания сенсоров на его основе [45]. Предложены различные сочетания ГКР-спектроскопии с методами разделения и концентрирования, например, газовой хроматографией [6], ТСX [7-8], жидкостной хроматографией и ВЭЖХ [9-12], капиллярным электрофорезом [13], жидкостной [14] и твердофазной экстракцией [15].

Для получения ГКР сигнала непосредственно на сорбенте необходимо введение в него наночастиц (НЧ) металлов, например, золота или серебра [16]. При этом нами использованы НЧ серебра как наиболее эффективные в плане увеличения сигнала ГКР и более экономичные [15]. В качестве сорбентов нами выбран оксид алюминия, как один из самых распространенных сорбционных материалов с хорошо разработанными методиками модификации для изменения сорбционных свойств. Так, поверхность оксида алюминия может быть модифицирована силанами, фосфоновыми, фосфиновыми и гидроксамовыми кислотами [17]. В работе [18] предложено модифицирование поверхности оксида алюминия пирокатехировым фиолетовым и тайроном (динатриевой солью 4,5-дигилрокси-м-бензолдисульфоновой кислоты) соответственно. В работе [19] в качестве модификатора был использован додецилсульфат натрия (ДДС) для определения гептафторбутановой, перфторгептановой, перфтороктановой кислот. В других работах ДДС использован для модификации алюмогелей в тандеме с другими веществами: 1-(2-пиридилазо) -2-нафтолом (ПАН) для определения кадмия и свинца в водных средах и растительных материалов [20]; 1,10-фенантролином для определения меди и кадмия в волосах человека и водных средах [21]; дитизоном на наночастицах магнетита с покрытием из окиси алюминия для определения ионов серебра в природных водах [22]. В этой работе для повышения гидрофобности алюмогеля проводили модификацию его поверхности ДДС.

Таким образом, целью данной работы явилось получение материалов на основе оксида алюминия, содержащих наночастицы серебра, а также изучение возможности модификации данных материалов с целью изменения их сорбционных свойств и использования для определения ПАУ методом ГКР. Введение НЧ серебра осуществляли непосредственно в процессе синтеза алюмогелей. В качестве модельного аналита для извлечения и последующего определения выбран представитель ПАУ - пирен. Использовали способ сорбционного концентрирования в статическом режиме, который обеспечивает равномерное распределение микрокомпонента по всему объему сорбента, что важно для последующего определения вещества.

\section{Эксперимент}

Материалы и реагенты. В работе использованы следующие реагенты и материалы: нитрат серебра (ACS reagent, Sigma-Aldrich), натрий лимоннокислый (ч.д.а., 
ГОСТ 5.1314-72), хлорид натрия (ACS reagent, Sigma-Aldrich), сульфат алюминия $\left(\mathrm{Al}_{2}\left(\mathrm{SO}_{4}\right)_{3} \cdot 18 \mathrm{H}_{2} \mathrm{O}, \geq 98 \%\right.$, Sigma-Aldrich), карбонат натрия $\left(\mathrm{Na}_{2} \mathrm{CO}_{3}\right.$, безводный, ACS reagent,), пирен (Sigma-Aldrich), додецилсульфат натрия (Sigma-Aldrich), азотная кислота (х.ч.).

Регистрацию ГКР-спектров проводили с использованием зондовой нанолаборатории Интегра Спектра со встроенным конфокальным микроскопом (объектив $100 \mathrm{x} / 0.9$ NA, Olympus, латеральное разрешение $\sim 1.5$ мкм) и спектрометром комбинационного рассеяния света (Solar). Определение пирена проводили в обращеннофазовом режиме ВЭЖХ с колонкой «Phenomenex» Luna 5UC18(2). В качестве подвижной фазы выступал раствор ацетонитрил : вода $(80: 20)$. Объем пробы составил 20 мкл, скорость потока $1 \mathrm{~cm}^{3} /$ мин. Размер НЧ серебра определяли методом сканирующей электронной микроскопии на приборе MiralLMU (Tescan, Великобритания) с использованием ускоряющего напряжения 15 кВ.

Синтез наночастиц серебра. Для синтеза НЧ серебра использовали цитратное восстановление серебра согласно [15,23]. 90 мг $\mathrm{AgNO}_{3}$ и 100 мг цитрата натрия добавляли к $500 \mathrm{~cm}^{3}$ кипящей воды. Смесь перемешивали при $95^{\circ} \mathrm{C}$ в течение 30 минут, затем охлаждали при комнатной температуре.

Синтез алюмогелей, содержащих НЧ серебра. Для получения оксида алюминия со встроенными НЧ серебра к $40 \mathrm{~cm}^{3}$ водного раствора активированных НЧ одновременно приливали $10 \mathrm{~cm}^{3} 0.3 \mathrm{M}$ раствора сульфата алюминия и $8 \mathrm{~cm}^{3} 0.4 \mathrm{M}$ раствора карбоната натрия при интенсивном перемешивании. Для активирования НЧ серебра проводили их предварительное искусственное агрегирование путем добавления $1.2 \mathrm{~cm}^{3}$ раствора хлорида натрия $(1.0 \mathrm{M})$ и последующем перемешивании в течение 10 секунд; активирование проводили непосредственно перед встраиванием НЧ с оксид алюминия. Для завершения реакции суспензию тщательно перемешивали в течение 5 мин на магнитной мешалке и центрифугировали (5 мин, 2000 об/мин). После отбора надосадочной жидкости, гель заливали деионизованной водой, обрабатывали зондовым У3 $(50 \% \mathrm{~W})$, снова центрифугировали, сливали надосадочный раствор и высушивали при $40-50^{\circ} \mathrm{C}$ в сушильном шкафу примерно в течении трех суток. После высыхания частицы застывшего геля растирали в фарфоровой ступке в присутствии этанола и затем снова высушивали.

Методика модифицирования алюмогелей. Модификацию алюмогелей проводили по методике, описанной в литературе [19]. Для этого 10 мг сорбента, помещали в пластиковые пробирки для микроцентрифугирования объемом $2 \mathrm{~cm}^{3}$, далее добавляли $0.5 \mathrm{~cm}^{3}$ ДДС с концентрациями $1.4 \cdot 10^{-2} \mathrm{M}$ и $2.7 \cdot 10^{-3} \mathrm{M}$ (в 0.01 н $\mathrm{HNO}_{3}$ или воде).

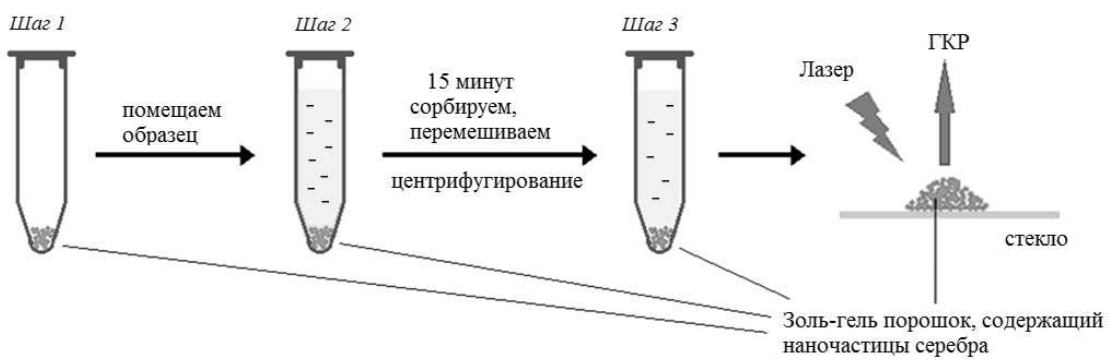

Рис. 1. Схема сорбционного концентрирования с последующим ГКР детектированием

Методика сорбционного концентрирования. Сорбцию пирена проводили в статических условиях. К навеске алюмогеля массой 10 мг, помещенной в пластиковую пробирку для микроцентрифугировани объемом $2 \mathrm{~cm}^{3}$, приливали раствор пирена (шаги 1 и 2 на рис. 1) и перемешивали смесь в течение 15 минут. После окончания 
сорбции смесь центрифугировали при 2000 об/мин в течение 3 минут и отделяли раствор от осадка.

Методика регистрации ГКР-спектров. Образец сорбента (10 мг) с сорбированным пиреном помещали на стандартное покровное стекло сразу же после отделения от надосадочной жидкости и проводили фокусировку лазерного луча на частицах оксида алюминия с помощью микроскопа и регистрировали спектры. Для получения усредненного результата с одного образца регистрировали от 20 до 30 спектров.

\section{Обсуждение результатов}

Синтез и характеризация НЧ серебра и алюмогелей с НЧ серебра. Получение НЧ серебра, покрытых цитратом, осуществляли согласно [23]. На рис. 2 приведено СЭМ изображение полученных НЧ, средний размер полученных НЧ составил $70 \pm 20$ нм. Для усиления сигнала ГКР проводили так называемое активирование раствора НЧ серебра непосредственно перед их встраиванием в оксид алюминия. Активирование включает в себя искусственное агрегирование НЧ путем повышения ионной силы раствора добавлением раствора хлорида. Показано, что повышение ионной силы с 10 до 60 ммоль/л приводит к увеличению размера НЧ серебра (определенного

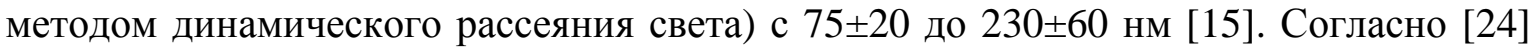
агрегированные НЧ Ag содержат много так называемых «горячих точек»- небольшие зазоры ( 1 нм) между НЧ. Электрическое поле в «горячих точках» значительно увеличивается под воздействием лазерного излучения, что обеспечивает более высокую интенсивность ГКР. В качестве оптимальной выбрана ионная сила равная 30 ммоль/л, обеспечивающая формирование димерных и тримерных агрегатов.

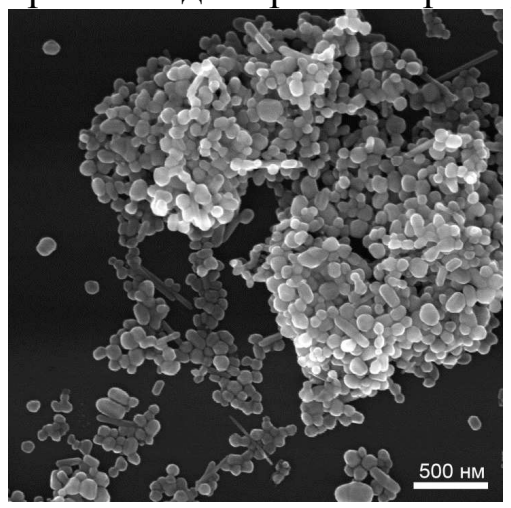

Рис. 2. СЭМ-изображение наночастиц серебра, полученных методом цитратного восстановления

Следующим этапом работы явилось получение материалов на основе оксида алюминия. В основе получения порошков на основе $\mathrm{Al}_{2} \mathrm{O}_{3}$ лежит реакция:

$$
\mathrm{Al}_{2}\left(\mathrm{SO}_{4}\right)_{3}+3 \mathrm{Na}_{2} \mathrm{CO}_{3}+6 \mathrm{H}_{2} \mathrm{O}=2 \mathrm{Al}(\mathrm{OH})_{3} \downarrow+3 \mathrm{Na}_{2} \mathrm{SO}_{4}+3 \mathrm{H}_{2} \mathrm{O}+3 \mathrm{CO}_{2} \uparrow
$$

Масса сорбентов составила $\sim 380$ мг $( \pm 5.3 \%)$ и выход реакции $\sim 250 \%\left(\mathrm{Al}_{2} \mathrm{O}_{3}\right)$, что указывает на большое количество кристаллизационной воды. Эта вода не может быть удалена путем сушки при высокой температуре, поскольку нагревание при температуре более $70^{\circ} \mathrm{C}$ приводит к уменьшению усиления комбинационного рассеяния. Оценка удельной поверхности и распределения пор по размерам показала, что сухие порошки $\mathrm{Al}_{2} \mathrm{O}_{3}$-HЧАg имеют два основных диапазона пор: от 1 до 10 нм, и от 10 до 50 нм с $20 \%$ и $80 \%$ вкладом соответственно, а удельная площадь поверхности составила $\sim 30 \mathrm{~m}^{2} / \Gamma$. 
Изучение сорбционных свойств алюмогелей с НЧ серебра. Далее изучали сорбцию пирена на полученном алюмогеле. Степени извлечения рассчитывали по отношению площадей пиков пирена на хроматограмме до и после сорбционного концентрирования (табл. 1). Из данных таблицы видно, что в диапазоне концентраций $1 \cdot 10^{-8}-1 \cdot 10^{-6}$ с увеличением концентрации пирена в целом происходит увеличение степени извлечения, однако она не превышает $68 \%$. Низкие значения степени извлечения объясняются гидрофильностью сорбента на основе алюмогеля.

Таблица 1. Степени извлечения пирена на оксиде алюминия со встроенными наночастицами серебра

\begin{tabular}{|c|c|c|c|}
\hline Концентрация, моль/дм & $\begin{array}{c}\text { Интенсивность } \\
\text { до сорбции }\end{array}$ & $\begin{array}{c}\text { Интенсивность } \\
\text { после сорбции }\end{array}$ & $\mathrm{R}, \%$ \\
\hline $1 \cdot 10^{-6}$ & 723.2 & 249.6 & 65.5 \\
\hline $5 \cdot 10^{-7}$ & 469.0 & 209.0 & 55.4 \\
\hline $1 \cdot 10^{-7}$ & 113.0 & 36.40 & 67.8 \\
\hline $5 \cdot 10^{-8}$ & 58.54 & 30.70 & 47.5 \\
\hline $1 \cdot 10^{-8}$ & 24.65 & 17.52 & 28.9 \\
\hline
\end{tabular}

Для повышения извлечения гидрофобного пирена проводили модификацию поверхности сорбента поверхностно-активным веществом (ПАВ). В качестве ПАВ использовали водные и кислотные растворы ДДС различной концентрации (табл. 2). Рассчитанные степени извлечения пирена из его водного $5 \cdot 10^{-7} \mathrm{M}$ раствора на модифицированных сорбентах приведены в таблице 2. Из данных табл. 2 видно, что модифицирование сорбентов ДДС приводит к значительному повышению степени извлечения пирена (до 30\%) по сравнению с немодифицированным сорбентом. При этом, чем больше концентрация ДДС, тем более высокие значения степени извлечения. Использование бидистиллированной воды, по сравнению с 0.01 н азотной кислотой, также повышает степень извлечения. Таким образом, оптимальными условиями для модифицирования сорбента является концентрация ДДС $1.4 \cdot 10^{-2} \mathrm{M}$ и использование чистой воды в качестве растворителя.

Таблица 2. Влияние модификации додецилсульфатом натрия (ДДС) оксида алюминия со встроенными наночастицами серебра на степень извлечения пирена $\left(5 \cdot 10^{-7} \mathrm{M}\right)$

\begin{tabular}{|c|c|}
\hline Состав модифицирующего раствора & $\mathrm{R}, \%$ \\
\hline ДДС $\left(1.4 \cdot 10^{-2} \mathrm{M}\right)+0.01 \mathrm{H} \mathrm{HNO}_{3}$ & 83.9 \\
\hline ДДС $\left(1.4 \cdot 10^{-2} \mathrm{M}\right)$ & 88.1 \\
\hline ДДС $\left(2.7 \cdot 10^{-3} \mathrm{M}\right)+0.01 \mathrm{H} \mathrm{HNO}_{3}$ & 78.1 \\
\hline ДДС $\left(2.7 \cdot 10^{-3} \mathrm{M}\right)$ & 85.2 \\
\hline Без модификации & 55.4 \\
\hline
\end{tabular}

При выбранных условиях получена изотерма сорбции пирена на алюмогеле, содержащем НЧ серебра, в интервале концентраций $10^{-6}-5 \cdot 10^{-4}$ моль/л. Показано, что изотерма сорбции подчиняется уравнению Фрейндлиха (рис. 3):

$$
a_{\mathrm{p}}=K_{\phi} \cdot C_{\mathrm{p}}^{1 / \mathrm{n}}
$$

где $a_{\mathrm{p}}-$ предельная сорбция, ммоль/г, $K_{\phi}$ - константа сорбционного равновесия. 


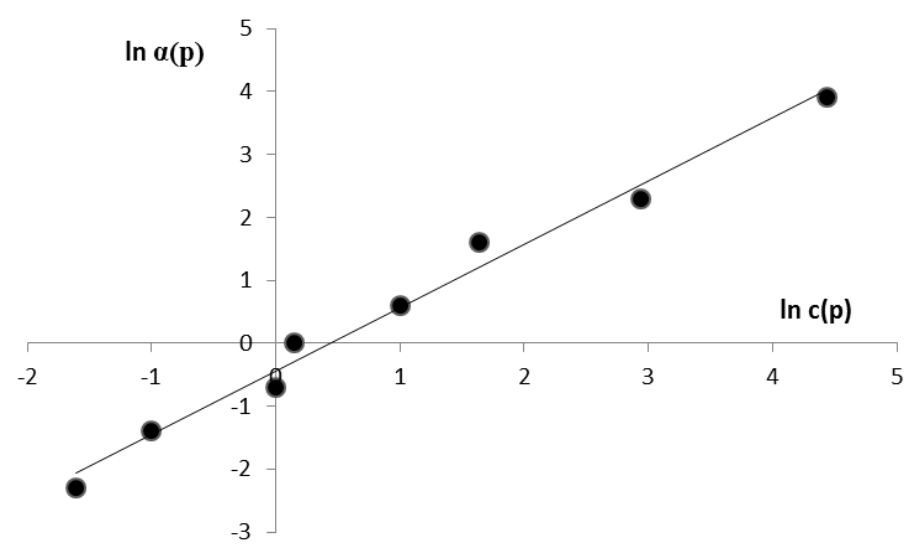

Рис. 3. Изотерма сорбции пирена в линейных координатах Фрейндлиха

Определение пирена методом ГКР-спектроскопии. Преимуществом полученных сорбентов и разработанной методики является то, что после проведения концентрирования пирена, его можно определить методом ГКР непосредственно в фазе сорбента. ГКР-спектр пирена, полученный после концентрирования его раствора с концентрацией $10^{-6} \mathrm{M}$ представлен на рис. 4. Поскольку ГКР-сигнал представляет собой характеристичный колебательный спектр, то сравнивая полученный ГКР спектр пирена с опубликованными ранее результатами [25] можно утверждать, что использование модификации с помощью ДДС не влияет на регистрируемый сигнал. Обнаружено, что погрешность измерения сигнала ГКР составляет от 5 до 22\%, что частично объясняется использованием в работе конфокальной оптической системы регистрации сигнала и 100x объектива, которые делают определение достаточно чувствительным к неоднородностям исследуемого образца.

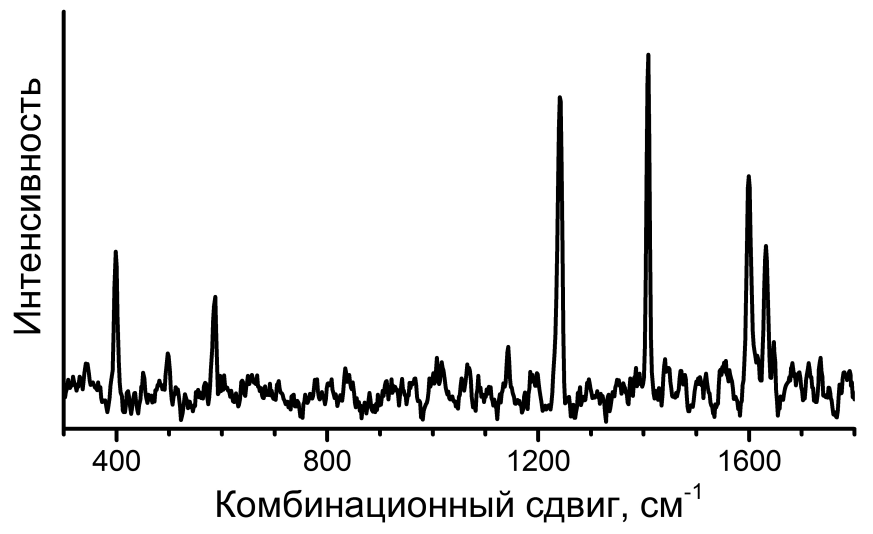

Рис. 4. ГКР-спектр пирена, сорбированного на алюмогеле из раствора с концентрацией $10^{-6} \mathrm{M}$

\section{Заключение}

В данной работе предложен подход к сочетанию сорбционного концентрирования пирена на алюмогеле, содержащем наночастицы серебра, с его последующим определением методом ГКР-спектроскопии непосредственно в фазе сорбента. Полученные методом цитратного восстановления наночастицы серебра предварительно агрегировали добавлением хлорида натрия и внедряли в алюмогель на стадии синтеза. Изучены сорбционные свойства полученных алюмогелей по отношению к пирену, и показана возможность увеличения степени извлечения путем модификации сорбента ДДС. Показано усиление КР-спектров пирена, сорбированного на полученном алюмогеле из $10^{-6} \mathrm{M}$ раствора. 


\section{Работа выполнена при поддержке Российского научного фонда, проект № 14-13-00229.}

\section{Список литературы}

1. Майстренко В.Н., Хамитов Р.3., Будников Г.К. Эколого-аналитический мониторинг суперэкотоксикантов. М. БИНОМ. Лаборатория знаний. 2004. 323 с.

2. Клименков В.Г., Борзенко А.Г. // Вестн. Моск. ун-та. Сер. 2. Химия. 2005. Т. 46. № 6. C. 392-394.

3. Цымбалюк К.К., Деньга Ю.М., Антонович В.П. // Методы и объекты химического анализа. 2013. Т. 8. № 2. С. 50-62.

4. Markin A.V., Markina N.E., Goryacheva I.Y. // TrAC. 2017. Vol. 88. pp. 185-197.

5. Markina N.E., Markin A.V., Zakharevich A.M. et al. // J. Nanopart. Res. 2016. Vol. 18. pp. 353-361.

6. Pieczonka N.P.W., Aroca R.F. // Chem. Soc. Rev. 2008. Vol. 3. pp. 946-954.

7. Freye Ch.E., Crane N.A., Kirchner T.B., Sepaniak M.J. // Analytical Chemistry. 2013. Vol. 85. No 8. pp. 3991-3998.

8. Chen J., Abell J., Huang Y.-W., Zhao Y. // Lab On A Chip. 2012. Vol. 12. No 17, pp. 30963102.

9. Carrillo-Carrión C., Simonet B.M., Valcárcel M., Lendl B. // J. Chromatogr. A. 2012. Vol. 1225, pp. 55-61.

10. Carrillo-Carrión C., Armenta S., Simonet B. M. et al. // Anal. Chem. 2011. Vol. 83. pp. 93919398.

11. Zachhuber B., Carrillo-Carrión C., Simonet Suau B.M., Lendl B. // J. Raman Spectrosc. 2012. Vol. 43. No 8. pp. 998-1002.

12. Lin M., He L., Awika J. et al. // J. Food Sci. 2008. Vol. 73. No 8, pp. 129-134.

13. Leopold N., Lendl B. // Anal. Bioanal. Chem. 2010. Vol. 396. pp. 2341-2348.

\section{References}

1. Majstrenko V.N., Hamitov R.Z., Budnikov G.K. Ekologo-analiticheskiy monitoring superekotoksikantov. M., BINOM: Laboratoriya znaniy, 2004, $323 \mathrm{p}$.

2. Klimenkov V.G., Borzenko A.G., Vestnik of Moscow University. Series 2. Chemistry, 2005, Vol. 46, No 6, pp. 392-394.

3. Cymbalyuk K.K., Den'ga Yu.M., Antonovich V.P., Methods and objects for chemical analysis, 2013, Vol.8, No 2, pp. 50-62.
14. Markina N.E., Shalabay V.V., Zakharevich A.M., Markin A.V. // Proc. of SPIE. 2016. Vol. 9917. 99170X.c

15. Yurova N.S., Markina N.E., Pozharov M.V et al. // Colloids Surf. A. 2016. Vol. 495. pp. 169-175.

16. Fang P.P., Lu X., Liu H., Tong Y. // TrAC. 2015. Vol. 66.pp. 103-112.

17. Лисичкин Г.В., Фадеев А.Ю., Сердан А.А. и др. Химия привитых поверхностных соединений. М. Изд-во ФИЗМАТЛИТ. 2003. $592 \mathrm{c}$.

18. Кубышев С.С., Тихомирова Т.И., Варламова Д.О. и др. // Вестник Моск. Ун-та, Сер.2. Химия. 2009. Т. 50 . № 2 . С. 104-108.

19. Zhao X., Li J., Shi Y. Et al. // J. Chromatogr. A. 2007. Vol. 1154. No 1-2. pp. 52-59.

20. Ezoddin M., Shemirani F., Abdi K. et al. // J. Hazardous Mater. 2010. Vol. 178. No 1-3. pp. 900-905.

21. Shabani A.M.H., Dadfarnia S., Dehghani Z. // Talanta. 2009. Vol. 79. No 4. pp. 1066-1070.

22. Karimi M.A., Hatefi-Mehrjardi A., Mohammadi S.Z. et al. // Int. J. Envir. Anal. Chem. 2012. Vol. 92. No 12. pp. 1325-1340.

23. Lee P.C., Meisel D. // J. Phys. Chem. 1982. Vol. 86. pp. 3391-3395.

24. Shiohara A., Wang Y., Liz-Marzán L.M. // J. Photochem. Photobiol. C. 2014. Vol. 21. pp. $2-25$.

25. Русанова Т.Ю., Маркин А.В., Юрова Н.С. и др. // Известия Саратовск. ун-та. Новая сер. Сер. Химия. Биология. Экология. 2013. Вып. 4. С. 12-18.

4. Markin A.V., Markina N.E., Goryacheva I.Y., TrAC, 2017, Vol. 88, pp. 185-197.

5. Markina N.E., Markin A.V., Zakharevich A.M. et al., J. Nanopart. Res., 2016, Vol. 18, pp. 353-361.

6. Pieczonka N.P.W., Aroca R.F., Chem. Soc. Rev., 2008, Vol. 37, pp. 946-954.

7. Freye Ch.E., Crane N.A., Kirchner T.B., Sepaniak M.J., Analytical Chemistry, 2013, Vol. 85, No 8, pp. 3991-3998. 
8. Chen J., Abell J., Huang Y.-W., Zhao Y., Lab On A Chip, 2012, Vol. 12, No 17, pp. 30963102.

9. Carrillo-Carrión C., Simonet B.M., Valcárcel M., Lendl B., J. Chromatogr. A, 2012, Vol. 1225, pp. 55-61.

10. Carrillo-Carrión C., Armenta S., Simonet B. M. et al., Anal. Chem., 2011, Vol. 83, pp. 9391-9398.

11. Zachhuber B., Carrillo-Carrión C., Simonet Suau B.M., Lendl B., J. Raman Spectrosc., 2012, Vol. 43, No 8, pp. 998-1002.

12. Lin M., He L., Awika J. et al., J. Food Sci., 2008, Vol. 73, No 8, pp. 129-134.

13. Leopold N., Lendl B., Anal. Bioanal. Chem., 2010, Vol. 396, pp. 2341-2348.

14. Markina N.E., Shalabay V.V., Zakharevich A.M., Markin A.V., Proc. of SPIE, 2016, Vol. 9917, 99170X.

15. Yurova N.S., Markina N.E., Pozharov M.V. et al., Colloids Surf. A, 2016, Vol. 495, pp. $169-175$.

16. Fang P.P., Lu X., Liu H., Tong Y., TrAC, 2015, Vol. 66, pp. 103-112.

17. Lisichkin G.V., Fadeev A.YU., Serdan A.A. et al., Himiya privityh poverkhnostnyh soedineniy, M., 2003, izd-vo fizmatlit, 592 p.

Юрова Надежда Сергеевна - аспирант кафедры аналитической химии и химической экологии, Саратовский национальный исследовательский государственный университет имени Н.Г. Чернышевского, Саратов

Захаревич Андрей Михайлович - зав. лабораторией диагностики наноматериалов и структур, к.ф.-м.н., Саратовский национальный исследовательский государственный университет имени Н.Г. Чернышевского, г. Саратов

Маркин Алексей Викторович - доцент кафедры общей и неорганической химии, к.х.н., Саратовский национальный исследовательский государственный университет имени Н.Г. Чернышевского, г. Саратов

Русанова Татьяна Юрьевна - зав. кафедрой аналитической химии и химической экологии, д.х.н., Саратовский национальный исследовательский государственный университет имени Н.Г. Чернышевского, г. Саратов
18. Kubyshev S.S., Tihomirova T.I., Varlamova D.O., Ivanov A.V., Nesterenko P.N., Vestnik of Moscow University. Series 2. Chemistry, 2009, Vol. 50, No 2, pp. 104-108.

19. Zhao X., Li J., Shi Y. Et al., J. Chromatogr. A, 2007, Vol. 1154, No 1-2, pp. 52-59.

20. Ezoddin M., Shemirani F., Abdi K. et al., J. Hazardous Mater., 2010, Vol. 178, No. 13, pp. 900-905.

21. Shabani A.M.H., Dadfarnia S., Dehghani Z., Talanta, 2009, Vol. 79, No 4, pp. 1066-1070.

22. Karimi M.A., Hatefi-Mehrjardi A., Mohammadi S.Z. et al., Int. J. Envir. Anal. Chem., 2012, Vol. 92, No 12, pp. 1325-1340.

23. Lee P.C., Meisel D., J. Phys. Chem., 1982, Vol. 86, pp. 3391-3395.

24. Shiohara A., Wang Y., Liz-Marzán L.M., J. Photochem. Photobiol. C, 2014, Vol. 21, pp. 2-25.

25. Rusanova T.Yu., Markin A.V., Yurova N.S. et al., Izvesiya Saratovsk. un-ta. Nov. Ser. Ser. Himiya. Biologiya. Ekologiya, 2013, No 4, pp. 12-18.

Yurova Nadezhda S. - the postgraduate student, department of analytical chemistry and chemical ecology, Saratov State University, Saratov, e-mail: nad4883@yandex.ru

Zaharevich Andrei M. - Ph.D. (physics and mathematics), the head of nanomaterials and structures diagnostic laboratory, Saratov State University, Saratov, email:

ZaharevichAM@yandex.ru

Markin Alexei V. - Ph.D. (chemistry), associate prof., department of common and inorganic chemistry, Saratov State University, Saratov, e-mail: av_markin@mail.ru

Rusanova Tatiana Yu. - grand Ph.D (chemistry), the head of analytical chemistry and chemical ecology department, Saratov State University, Saratov, e-mail: tatyanarys@yandex.ru 